Christopher Paul Szabo ${ }^{1 *}$

\title{
Eating disorders, risk and management: a personal journey and a South African and African perspective
}

\author{
'Department of Psychiatry, Faculty of Health Sciences, University of the Witwatersrand, Johannesburg, South Africa \\ *email: Christopher.szabolawits.ac.za
}

DOI: 10.2478/gp-2019-0017

Received: 1 May 2019; Accepted: 9 August 2019

\begin{abstract}
Eating disorders are amongst the most, disabling and lethal of psychiatric conditions. These conditions affect predominantly adolescent and young adult females, but not exclusively so. Whilst stereotypically diagnosed in western, urban societies their emergence in a range of population groups beyond the aforementioned has challenged the stereotype - no more so than in South Africa where eating disorders had not been diagnosed in black, female South Africans until the 1990s. Creating awareness of the changing demographic profile and thus risk was critical in ensuring appropriate diagnosis and management. Whilst this was a key feature of my personal journey there were numerous other aspects of risk explored for the development of eating disorders, as well as management, which will be highlighted and discussed in the paper. This has been especially relevant within the South African context.
\end{abstract}

\section{Keywords}

eating disorders, South Africa, post- apartheid, Africa, risk, management

\section{INTRODUCTION}

Eating disorders have been documented in South Africa since the 1970s with the earliest descriptions of clinical samples emanating from both Cape Town and Johannesburg (Nash \& Colbon, 1994; Beumont, George \& Smart, 1976; Norris, 1979; Touyz \& Beumont, 1984). These conditions, at that time, conformed demographically to the prevailing stereotype i.e. young, white females. The 1980s brought a series of publications, from African countries, of case reports of eating disorders thus dispelling the notion that these conditions afflict only white females in urban, "western" settings (Nweafuna, 1981; Buchan \& Gregory, 1984; Famuyiwa 1988; Ilechukwu \& Nhiwatiwa, 1988; Fahy, Robinson, Russell \& Sheinman, 1988). The 1990s heralded change and is where the origins of this individual journey emanate.

The content that provides "the story" represents selected publications related to my work in eating disorders covering a period of over 20 years (1995-2016). The nature of the publications is diverse. However, 2 clear dimensions emerge, risk and management. This started with the description of an emerging phenomenon and thereafter attempting to quantify future risk as a public health issue, with implications for diagnosis (through awareness) and resources. The works also speak to factors contributing to risk e.g. culture, gender, dieting, religion etc. as well as describing aspects of treatment in relation to hospitalized patients which included both review of outcomes, novel interventions and complications. The most recent contribution (a chapter) related to an area of work that spoke to my research and consequent data/ publications i.e. documenting the emergence of eating disorders amongst black, female South Africans with the subsequent community based work to determine risk (Szabo, 2015) . Specifically the chapter related to eating disorders in so called underrepresented countries with the content covering not only Africa but also South America, Asia and the Middle East and providing a comprehensive analysis of what has emerged in these regions. Aside from the trends observed for research/published content emanating from specific regions, a key conceptual understanding was highlighted. Specifically that the understanding of culture bound as proposed by Lee (1996) which constituted a profound re-conceptualization of the term, with global implications. Of note was that this reconceptualization had arisen as a consequence of the emergence of eating disorders where previously they were thought not to exist i.e. the developing world, the so called under-represented countries. Specifically that eating disorders were bound to 
the culture of modernity which Lee (1996) defined and will be elucidated later in this paper.

Having completed specialist training in 1992 I was appointed as a consultant in the eating disorders and adolescent unit at Tara Hospital in 1993 (Tara Hospital is a specialized psychiatric hospital affiliated to the University of the Witwatersrand's Department of Psychiatry, located in Johannesburg, South Africa). The country was on the verge of entering the post - apartheid era. More specifically, the then Apartheid Government having announced the unbanning of the African National Congress years earlier with the subsequent release of their leader - Nelson Mandela - in 1990 after 27 years of imprisonment ultimately saw him lead the country (following the first democratic elections which took place in 1994). Immersing myself in clinical work as I sought to acquire the specific knowledge and skills required to effectively manage this highly select group of patients i.e. those of all ages with eating disorders (as well as the adolescent group who suffered from not only eating disorders but the full spectrum of psychopathology) I became conscious of the possibility that with regard to eating disorders it was surely inevitable that the demographics would change. Indeed they did, and this is where my journey as a clinician/researcher began. Whilst my career path took me away from Tara in 2004, I continued to work in the field of eating disorders and during a period of over 20 years (1995 - 2016) published, as author or co-author, over 40 articles related to eating disorders. These publications covered the full spectrum of publications from original research to case reports, opinions, editorials and chapters. Of all the publications there is one that stands out - the paper that documented the first reported and published cases of eating disorders in black, South African females (Szabo, Berk, Tlou \& Allwood, 1995). Some of the published work emanated from a range of higher degrees undertaken under my supervision at a Masters $(\mathrm{n}=7)$ and $\mathrm{PhD}$ level $(\mathrm{n}=1)$ noting publications from my own $\mathrm{PhD}$ related to eating disorders which are included and referenced. The supervised Masters work took place predominantly at Tara but also involved community samples - as was the case for the $\mathrm{PhD}$ work.

Following my time at Tara, my career path took me into senior hospital and academic management with my appointments as Head of Clinical Department (Psychiatry) at Charlotte Maxeke Johannesburg Academic Hospital and Academic Head of Department of Psychiatry in the Faculty of Health Sciences at the University of the Witwatersrand in Johannesburg, South Africa. My role changed significantly, with a more general focus in terms of clinical Psychiatry and the responsibilities of leadership. However, eating disorders remained a focus notwithstanding change and I continued to provide supervision and guidance to the eating disorders unit as well as work clinically with eating disordered patients. Subsequent to my leaving Tara the eating disorders unit continued to render an important in patient service with a multi-disciplinary approach - and continues to do so. In addition, the hospital provides comprehensive in and out- patient services for adult, adolescent and child patient populations.

The content that follows is an attempt to provide a coherent narrative that links the work, and demonstrates a contribution to the local, national and international literature and details my journey both clinically and in terms of related research. When reviewing the body of this work it became apparent that there were two predominant themes that could be used to characterize the work i.e. risk and management, hence the title of the paper. In terms of risk, this denotes factors potentially contributing to the risk of developing an eating disorder. The content has thus been structured in accordance with this understanding. More specifically, whilst I will provide a specific focus on my work in the field of eating disorders within a South African and African setting it should be noted that reference - in the current paper - is made to related work from within the South African and African settings as well as internationally, that had direct reference to this work, as context. However, comprehensive reviews of eating disorders in Africa have been provided in both a book on eating disorders - written by myself - in 2009 (Szabo, 2009) as well as chapters in books in 2011 (Szabo, 2011) and 2015 (Szabo, 2015) with the 2011 book on African Psychiatry coedited by myself together with David Ndetei. Collectively these published works comprehensively detailed all published work related to eating disorders in Africa - that was available at the times of publication - which is beyond the focus of the current paper. A Pubmed search $\left(11^{\text {th }}\right.$ June 2019) using the terms eating disorders, African, South African yielded 72 citations, 20 of which were directly relevant with 9 of these articles involving myself. However, based on the aforementioned detailing of published work [2009; 2011; 2015] which goes significantly beyond the PubMed yield - the PubMed search highlights a key issue i.e. the under representation of content from Africa. It should also be emphasized that the current paper provides a perspective rather than an all- encompassing review.

\section{EMERGENCE: CASES}

The clinical diagnosis of eating disorders affecting black South African females (Szabo, Berk, Tlou \& Allwood, 1995) represented the emergence of what had been anticipated i.e. with the advent of societal change influenced by political change clinicians might begin to see young black females with eating 
disorders . At the time of publication of the aforementioned paper describing the first cases there had been no descriptions of eating disorders in this group of South Africans, clinically, in the published literature. The significance was that the documented existence of eating disorders in this population group challenged conventional demographic stereotypes of eating disorder sufferers insofar as it had been assumed that these conditions did not affect black, South African females. Further, it now sensitized clinicians to this diagnostic possibility amongst young black South African females. The question I asked myself was whether these cases constituted the forerunners of further such cases with potential implications for resource allocation i.e. what was the potential future clinical risk and were the current services adequately resourced to deal with an increase in demand? Beyond clinician implications, there were issues for the community at large in terms of illness recognition and appropriate help seeking i.e. would there be recognition of these conditions within the community and appropriate help seeking by sufferers, and more specifically would clinical staff be equipped to assist.

\section{ATTITUDES: COMMUNITY}

Given that in the early 1990's racial integration had commenced at school level, specifically private schools, it seemed that western, urban value systems to which young black South African females might have been directly exposed rendered this setting appropriate for researching the question related to potential future risk (Szabo \& Hollands, 1997a). Proximity to western culture and risk for developing eating disorders had been explored in an earlier Zimbabwean study of black and white school girls at a secondary school in Harare, which served as background in terms of approach (Hooper \& Garner, 1986). The study by Szabo and Hollands (1997a) was undertaken at an all girls, racially diverse private school located in Johannesburg in the mid 1990s. The study (Szabo \& Hollands,1997a) made use of the 26 item version of the Eating Attitudes Test (EAT - 26), a self-report questionnaire which measures the existence of attitudes that may reflect current/pre or subclinical eating disorders. Whilst well established as a screening tool internationally the EAT -26 had not been validated in a South African population with the study seemingly the first to use the instrument in a South African population. The study yielded a suprising finding insofar as establishing that the prevalence of abnormal eating attitudes was greater in black pupils but where there were abnormal eating attitudes the profile of the abnormality was not different for the black and white samples. The former finding stood in contrast to the earlier Zimbabwean study (Hooper \& Garner, 1986) in that those researchers had found that the white pupils were more likely to demonstrate a desire for thinness and related dieting behaviour - noting that the study had been conducted 9 years earlier and had used the Eating Disorders Inventory (EDI). Further, that the "drive for thinness" subscale of the EDI has a relatively high correlation with the "dieting" subscale of the EAT 26, a key factor in the development of eating disorders as will be discussed later. However, the latter finding by Szabo and Hollands (1997a) of similarity in profile between race groups where abnormal eating attitudes were documented was congruent with the earlier clinical findings reported by Szabo, Berk, Tlou \& Allwood (1995) i.e. race did not appear to impact on clinical presentation. This sentiment was further expressed in a publication noting that the number of cases of black eating disorder sufferers Szabo had been clinically involved with had subsequently increased to nine (Szabo, 1999), since the first description of such cases (Szabo, Berk, Tlou \& Allwood, 1995) , with the ..."absence of any unique features designating the sufferer as black." (Szabo, 1999).

Of significance was the extent of abnormal eating attitudes amongst black pupils (Szabo \& Hollands, 1997a) which was comparable to findings amongst very high risk groups e.g. immigrant populations under acculturative stress, both in the UK and the USA Mumford, Whitehouse \& Platts, 1991; Pumariega, 1986) as well as a South African sample of ballet dancers (le Grange, Tibbs \& Noakes, 1994). The implication was one of future risk for cases to emerge from within the black community.

The initial published work on eating attitudes (Szabo \& Hollands, 1997a) served as the basis for my own $\mathrm{PhD}$ work. Publications emanating from the $\mathrm{PhD}$ documented the extent of abnormal eating attitudes in urban and rural samples Szabo \& Allwood, 2004a). A specific component of the $\mathrm{PhD}$ involved the translation and validation of a Zulu version of the EAT -26 (Szabo \& Allwood, 2004b). A further component of the PhD related to body figure preference across racial groups in the different settings (Szabo \& Allwood, 2006). The key finding in terms of eating attitudes, as measured by the Eating Attitudes Test (EAT-26), was that the extent of abnormal attitudes was equivalent amongst white and black urban high school students i.e. $18.6 \%$ (Szabo \& Allwood, 2004a) with a notable reduction amongst black, rural high school students with a prevalence of 3\% (Szabo \& Allwood, 2004a). This finding appeared to confirm the hypothesis that urban dwellers were at greater risk, within a South African setting. A further key finding based on the Body Figure Preference data demonstrated fewer rural students wanting to be smaller compared to urban students, with the potential drive to be thinner least amongst rural, black students (Szabo \& Allwood, 2006). These findings thus 
suggested a role for milieu - more than race - which was to be echoed in subsequent work.

Subsequent research sought to extend Szabo's $\mathrm{PhD}$ work by studying more directly the phenomenon of exposure to western culture with a specific emphasis on the phenomenon of acculturation as a risk factor for the emergence of eating disorders amongst black, female, South African adolescents (Morris \& Szabo, 2013). The research - towards a PhD supervised by Szabo - was undertaken in Kwa Zulu Natal at 5 schools with varying degrees of racial integration/profile ranging from $100 \%$ black to $13 \%$ black. Kwa Zulu Natal is one of South Africa's nine provinces with a predominant Zulu population. Four of the schools were selected for the Focus Group/qualitative component of the research leading to the subsequent publication (Morris \& Szabo, 2013). Two of the selected schools comprised a black majority $(100 \%, 96 \%)$ and 2 comprising a black minority (39\% and 13\%) - relative to white pupils. The published study (Morris \& Szabo, 2013) being qualitative in approach provided a more nuanced understanding of meaning i.e. provided insights beyond quantitative data related to eating attitudes/behaviour. The study (Morris \& Szabo, 2013) was part of $\mathrm{PhD}$ work that involved administration of a range of instruments with a specific aim to establish the role of acculturative stress and the emergence of eating disorders. The PhD established that acculturative stress increased the risk of eating disorders, specifically where there was cultural identity confusion. A paper in this regard was indeed prepared but never published (neither by the candidate, nor by Szabo as supervisor).

The findings of the qualitative component of this $\mathrm{PhD}$ work (Morris \& Szabo, 2013) provided insight into the need for caution, clinically. Specifically as culturally sanctioned eating behaviour e.g. fasting, excess eating and purging might mask disordered eating behavior which may then go unrecognized as being symptomatic of an eating disorder. The finding was that pressures (western) towards thinness that might influence eating disordered behaviour certainly existed -as part of an eating disorder. However as culturally sanctioned eating practices may not be recognized as such, sufferers would potentially not receive the necessary clinical recognition or intervention.

\section{CLINICAL EXPERIENCE}

Aside from community based studies that attempted to infer risk from screening (Szabo \& Hollands, 1997a; Szabo \& Allwood, 2004a), a number of studies of clinical samples were undertaken involving data from the Eating Disorders Unit at
Tara Hospital (Gabriel \& Szabo, 2001; Delport \& Szabo, 2008). The shift in focus from community based, non-clinical, samples to clinical samples reflected how the initial case descriptions followed by studies to determine possible risk for further cases had in fact seen the emergence of such cases that were now being hospitalized - and thus the subjects of clinical research. It should be emphasized that the flow of research did not follow a specific programme as such but was influenced by how the clinical situation unfolded with dates of publications reflecting the time sequence.

The study by Gabriel and Szabo (2001) specifically focused on anorexia nervosa. In reviewing admitted cases over a 10 year period (1987 -1996), that included the publication of the first reported cases (Szabo, Berk, Tlou \& Allwood, 1995) they established that for the period of study the demographic profile of hospitalized anorexics conformed to the prevailing stereotype i.e. white, female, adolescent, middle - upper socio economic status. However, it was noted that hospitalized anorexics "...do not necessarily reflect the overall situation." i.e. clinically; further that black, eating disorder sufferers, whilst they exist, had not - during the period of review - found their way to a tertiary level, specialized eating disorders unit (Gabriel \& Szabo, 2001).This study served to confirm the demographic profile of hospitalized sufferers but with the potential for a changing profile given the emergence of eating disorders in the black, South African female population. In essence this was to sensitize clinicians of a potential future risk.

Subsequent to the study by Gabriel and Szabo (2001) a study by Delport and Szabo (2008) reported on psychometric data comparing black and white eating disorder sufferers who had been admitted to the eating disorders unit at Tara Hospital, confirming what had been suggested in the study by Gabriel and Szabo (2001) i.e. that there was a future risk [given that the study by Delport and Szabo (2008) followed the one of Gabriel and Szabo (2001)]. A total of 13 black female sufferers had been admitted to the eating disorders unit for the period 1998- 2004, with 7 diagnosed with anorexia nervosa and 6 with bulimia nervosa. Adequate data were available for 5 from either diagnostic group. The study sample of Delport and Szabo (2008) thus comprised both anorexia nervosa and bulimia nervosa sufferers, with age matched samples of anorexics and bulimics from either race group (black; white). The finding of this study (Delport \& Szabo, 2008), in keeping with earlier clinical observations (Szabo, Berk, Tlou \& Allwood, 1995), was that no differences were found on a range of questionnaires measuring both eating disorder and mood symptoms. Not only did this study (Delport \& Szabo, 2008) provide the first South African inter-ethnic data on eating disorders but it also 
challenged the notion of differences based on race that had been noted internationally - albeit with an increasing understanding that there were indeed more similarities than differences.

\section{SOUTH AFRICA - IMPLICATIONS FOR A SOCIETY IN TRANSITION}

At the time of the emergence of eating disorders amongst black South African females, the questions raised were "why" as in why might this emergence of such conditions be taking place and "why now" as in the 1990s?

\section{WHY?}

In an article I had written dealing with cross-cultural aspects of eating disorders (Szabo, 1998a) it was emphasized that the conceptualization of eating disorders as "culture bound" may well need to be reconsidered. Explicit was that such conditions had been traditionally "bound" to contemporary, western, urban culture albeit potentially "... because of the paucity of literature from non-western sources...” (Szabo, 1998a). Of importance to South African clinicians was the idea that race was less a factor in the development of an eating disorder than milieu i.e. if certain cultural factors existed e.g. values associated within a contemporary, urban setting, race was not a mitigating factor. Political change in South Africa had led to social change, with urbanization in particular. Of specific relevance was the observation, generally, of black sufferers to manifest bulimic symptoms (Dolan, 1991), noting that bulimic symptoms amongst eating disordered patients had been linked to urbanization (Hoek, Bartelds, Bosveld, van der Graff, Limpens, Maiwald, \& Spaaij, 1995). Hence socio-political change was understood to be implicated in the emergence of eating disorders amongst black, South African women.

\section{WHY NOW ?}

One of the theories regarding the emergence of eating disorders in societies that were not typically associated with such conditions was that of cultures in transition. These questions informed the basis of a chapter in a book on eating disorders and societies in transition in relation to South Africa (Szabo \& le Grange, 2001). The timing - mid 1990s - of the emergence of eating disorders amongst black, South African, women coincided with dramatic political change that defined South Africa as a post-apartheid state. A number of key background issues were identified that could have explained the absence of described cases prior to those by Szabo, Berk, Tlou and Allwood (1995) et al, namely the idea of eating disorders as a white problem (Szabo \& le Grange, 2001) as well as the role of traditional healers in dealing with eating related problems that were understood as culturally driven and therefore did not present to the establishment medical system (Szabo \& le Grange, 2001) - as noted by Morris and Szabo (2013) years later. The chapter explored the basis of a possible struggle with identity that could explain the risk amongst black, South African, women. It had been suggested that evolving emancipation (Szabo \& le Grange, 2001) as a consequence of political change, together with embracing a new found pride in being African (Szabo \& le Grange, 2001) as a consequence of political change contributed to internal psychological turmoil and the emergence of eating disorders i.e. the consequence of conflicted identity with the body as a ready vehicle for the expression of such conflict.

Of interest, Szabo and le Grange (2001) did not refer to work by Lee (1996) who in a seminal paper based on his experience of the emergence of eating disorders amongst Chinese in Hong Kong had re-evaluated the meaning of "culture bound" (Lee, 1996). Lee (1996) re-conceptualized "culture-bound", describing this reconceptualization as “...trans-national and value driven across geographical locations rather than unique to locations i.e. within the culture of "modernity". Such a culture was proposed to be comprised of a number of components which include "...industrial capitalism, urbanization, immigration, abundance of food, rising population weight, advanced information technology, proliferation of body orientated advertisements, decreased birth rate and changing roles of women.". In essence, it could have been that political change opened the door for "modernization" which may well have been the driving force of the emergence noted by Szabo and le Grange (2001), as had been observed in other countries shifting from traditional to modern.

\section{FACTORS INFLUENCING EATING ATTITUDES}

The study by Szabo and Hollands (1997a) to determine the extent of abnormal eating attitudes in a high school population was undertaken simultaneously with a study to determine factors associated with abnormal eating attitudes that might inform preventive strategies within a comprehensive treatment approach (Szabo \& Hollands, 1997b). A number of interesting findings emerged, albeit from a select sample, specifically that weight concerns whilst not exclusively associated with abnormal eating attitudes were an almost universal phenomenon within the sample (Szabo \& Hollands, 1997b). More specifically it was perception of weight rather than actual weight which predicted higher EAT-26 scores. Moreover, the existence of a history of dieting was extensive. Specific behaviours such as weighing as well as topics of conversation e.g. about food and dieting 
predicted higher EAT-26 scores and were thus highlighted as possible indicators of risk. The role of mothers in terms of their eating behaviour as well as their perceptions of daughter's behaviour was also highlighted. Finally, encouragement to lose weight was also identified as a risk factor for higher EAT-26 scores highlighting a need for sensitivity in this regard. Findings from the study thus provided evidence of a range of factors with practical implications in both detection and prevention. No prior South African study had done so.

\section{GENDER - THE EXISTENCE OF MALE SUFFERERS}

Eating disorders are generally understood to stereotypically be female conditions, with cases amongst males rare. However, a number of males had indeed been admitted to Tara Hospital's eating disorders unit. Within the South African context there was no such data. A paper by Freeman and Szabo (2005) comprised a review of clinical data of 17 male eating disordered patients admitted for treatment over a period of 10 years $(1993$ - 2002). The majority were diagnosed with anorexia nervosa (76\%). Notable findings included a predominance of upper socio economic status, with prominent exercise activity associated with the eating disorder. Use of the Eating Disorders Inventory to track progress over the course of hospitalization demonstrated what appeared to be worsening scores at discharge compared to admission albeit with an increase in body mass index over the course of admission. This finding suggested that the period of admission was potentially too short i.e. the physical response was not matched by psychological adaptation to such required change. Moreover, associated clinical issues were somewhat different to the international literature. Specifically it was found that mood and obsessive- compulsive disorders were infrequent but relational difficulties were common in the study by Freeman and Szabo (2005), notably father -son issues. This study thus provided some unique insights, involving a South African patient population.

Whilst the paper by Freeman and Szabo (2005) provided data on clinical cases, an earlier opinion piece had appeared in Playboy magazine on the subject of male eating disorders (Szabo, 1994). Written as an opinion piece, in the Health and Fitness section, it was a direct caution to men and an attempt to sensitize. More specifically the article introduced the concept of "reverse anorexia" whereby men perceive themselves to be too small and thus exercise excessively to increase body size with potential progression to anabolic steroid abuse. In time "reverse anorexia" became known as "muscle dysmorphia" and was described in a South African sample (Hitzroth, Wessels, Zungu-Dirwayi, Ooosthuizen \& Stein, 2001).
It was a clinical review of a male eating disordered patient, 22 years after initial diagnosis at Tara Hospital's eating disorders unit, that highlighted a critical issue i.e. the distinction between disordered eating as a harbinger of a neurological condition versus a primary eating disorder (Szabo, 2008). The patient in question had features suggestive of an eating disorder i.e. weight loss and purging but denied a drive for thinness or that the vomiting was self-induced. In subsequent years he was diagnosed with germinomas (frontal and cerebellar) which were treated with neurosurgery and radio therapy with consequent weight restoration and cessation of vomiting. This highlighted a key issue that eating disorders in males are generally rare, and that clinicians need to be sensitive to a misdiagnosis of an apparent eating disorder.

\section{RELIGION - EXPLORING RELEVANCE}

Whilst religious affiliation had not been definitively associated with eating disorders, the literature had demonstrated that female Jewish adolescents and young women were more likely to demonstrate abnormal eating attitudes/behaviours than their non-Jewish counterparts (Sykes, Leuser, Melia \& Gross, 1988; Kim, 2007; Pinhas, Heinmann, Bryden, Bradley, \& Toner, 2008). Given this finding it was decided to explore this within a South African setting as this had not been undertaken previously.

A study by Visser, Notelovitz, Szabo and Fredericks (2014) was the first to document abnormal eating attitudes amongst Jewish girls attending a traditional Jewish school, in South Africa. Importantly the sample comprised not only the learners but also their teachers. Rates of abnormal eating attitudes and related behaviours within the sample were comparable to relevant international and South African data derived from adolescents generally, albeit within the upper range. Most teachers at the school underestimated the extent of such issues and whilst acknowledging the need to address such issues they did not feel it appropriate within the school setting. Whilst not specifically demonstrating increased risk the data highlighted risk and also identified that within a school setting teachers needed to be appropriately sensitized to recognition and equipped to intervene at a level that did not interfere with their primary role.

\section{PERFECTIONISM}

Premorbid personality traits as a risk factor for eating disorders, specifically anorexia nervosa, had been the subject of speculation. A study by Srinivasagam, Kaye, and Plotnicov, Greeno, Weltzin and Rao (1995) had noted that following 
weight restoration and one year of sustained recovery from anorexia nervosa, the trait of perfectionism remained. This suggested that perfectionism may well be a trait predisposing to development of the condition rather than a state mediated phenomenon. Szabo and Terre Blanche (1997) responded to the study with evidence supporting the finding from their own work involving measurement of "perfectionism" using the Eating Disorders Inventory. Szabo and Terre Blanche (1997) demonstrated that scores on this subscale of the self - report questionnaire remained stable despite recovery and discharge with all other subscale scores demonstrating significant reduction.

\section{FAMILY}

The role of the family in the pathogenesis of eating disorders had been a subject of debate ranging from directly causative (Selvini Palazzoli, 1974; Minuchin, Rosman \& Baker, 1978) to being contested insofar as there being no specific family environment for either anorexia or bulimia nervosa (Dare, Le Grange, Eisler \& Rutherford, 1994). Research comparing family functioning between anorexia nervosa and cystic fibrosis sufferers as well as psychiatric and community controls revealed no differences (Blair, Freeman \& Cull, 1995). Against this background, Szabo, Goldin and Le Grange (1999) employed the Family Relations Scale in a sample of 17 eating disordered patients and their families, as well as 20 non psychiatric controls and their families. Whilst finding general similarities, as noted by Blair, Freeman and Cull (1995) there were also differences that suggested implications for intervention. For anorexic families there was a perception of lack of hierarchy, specifically noted by fathers but also experienced by the patients. For bulimic families the fathers experienced their families as less flexible, with the patient experiencing the family as tolerant of disagreement. The suggestion was that disagreement as experienced by the daughter was unresolved due to rigidity as experienced by the father. For either condition, there were father-daughter perceptions that suggested a need for greater awareness of the father - daughter dynamic in eating disordered families as opposed to the traditional approach of emphasizing the mother - daughter relationship (Pike \& Rodin, 1991). Such a finding had not previously been documented in a South African eating disordered sample, and provided an important clinical insight in terms of the need to consider the role of fathers and their involvement in treatment.

\section{MEDIA \& DIETING}

The role of the media has long been implicated in the pathogenesis of eating disorders, specifically within the context of promoting certain stereotypes of beauty and contributing to body dissatisfaction and dieting (Field, Chueng, Wolf \& Herzog , Gortmaker, \& Colditz, 1999). Other research has noted that such exposure impacts more specifically on those with an underlying eating disorder or body dissatisfaction Hamilton \& Waller, 1993; Cameron \& Ferraro, 2004) . Yet it was the earlier work of Garner, Garfinkel and Schwarz et al $(1980)^{48}$, that served as the basis for the study by Szabo (1996) on Playboy centrefolds. In the study by Garner, Garfinkel and Schwarz et al (1980) a potential basis for body dissatisfaction amongst women was proposed. They studied the discrepancy between Playboy centrefold and Miss America beauty pageant body dimensions relative to standard insurance table data for body dimensions of women over a corresponding period. The former had gotten smaller and the latter, larger. Hence there was a "real - ideal" discrepancy serving as a basis for body dissatisfaction, dieting, and increasing rates of eating disorders. Szabo (1996) undertook a review of Playboy centrefold weight and height data for 12 consecutive issues of the then recently unbanned version that had become available in South Africa (February 1994 - February 1995). From this data the Body Mass Index (BMI; weight in kilogrammes divided by height in metres, squared) of each centrefold was calculated - with $72 \%$ having a BMI of less than 18 i.e. technically underweight. This was clearly not so on inspection. What was understood was that such data potentially created the erroneous perception of the desirability of being underweight, with consequent impact on vulnerable individuals and the promotion of disturbed attitudes, behaviours and possible eating disorders. The finding emphasized a need for improved "media literacy". This was not a theme that had been explored in this way in a South African context, and it highlighted an important consideration when developing prevention strategies.

With regard to dieting - as mentioned earlier - and with so much emphasis on risk taking behaviour in adolescence e.g. unprotected sex, drug use etc. dieting had not been identified as one such behavior (Szabo, 2002). Noting the role of dieting in the aetiology of eating disorders was critical, and this served as the basis for an editorial by Szabo (2002), given the extent of adolescent dieting - from both international (Paton, Carlin \& Shao, Hibbert, Rosier, Selzer, \& Bowes, 1997) and South African data (Szabo, 2002). This was against a background of the promotion and existence of dieting in the general population. A specific point of concern was that most dieting was not indicated for health reasons (Paton, Carlin \& Shao, Hibbert, Rosier, Selzer, \& Bowes, 1997). The editorial then highlighted the consequent impact of eating disorders, not only on quality of life but also with anorexia nervosa having the highest mortality of all psychiatric conditions. The issue was thus raised with 
a call for consideration of dieting to be included amongst risk factors for adolescent health (Szabo, 2002). Within the context of adolescent risk taking behavior, such a consideration had not been proposed previously.

\section{NEUROTRANSMITTER FUNCTIONING}

Whilst eating disorder aetiology was generally understood to be socio- culturally driven, the role of biology in relation to neurotransmitter function was also a focus of research. A specific neurotransmitter was serotonin given its role in appetite control. The question was whether any such changes were state or trait related (Wolfe, Metzger \& Jimerson, 1997). Research into serotonergic functioning amongst anorexia and bulimia nervosa sufferers, compared to non-eating disordered controls, conducted by Berk, Kessa, Szabo and Butkow (1997) was the first such data on a South African eating disordered population. The key finding was that disturbed serotonergic functioning was only established for those anorexics with subsyndromal depression i.e. it was related to mood rather than an eating disorder. The suggestion was thus that such alterations in serotonergic functioning were more likely to be state related and potentially nutritionally based. Such research was novel in a South African setting given that it focused on biological aspects of eating disordered patients, and provided an understanding that underpinned a clinical approach. Specifically, and as will be noted in the section on Management, the presence of depressive features does not necessarily indicate a need for anti -depressant medication

\section{MANAGEMENT AND TREATMENT}

One of the major issues in the management of eating disordered patients is the reluctance of mental health professionals to involve themselves therapeutically i.e. not wanting to treat eating disordered patients. This is likely as much to do with the patient's resistance to treatment, and perceived likelihood of poor outcome, as it has to do with professional's lack of knowledge and experience. Specifically that management is best undertaken at a specialist level and preferably by a professional with relevant experience. Such professionals are in short supply. To this end the need for promoting awareness as well as providing guidance to health professionals generally and mental health professionals specifically resulted in a number of South African publications written by Szabo with that in mind (Szabo, 1995; Szabo, 2010). Whilst many psychiatric conditions have psychopharmacological intervention as a primary treatment modality, eating disorders generally have psycho-social interventions as the primary treatment modality - with an emphasis on cognitive-behaviour therapy.
The content on psychopharmacological interventions (Szabo, 1995; Szabo, 2010) emphasized the predominantly adjunctive use of medication for symptom based treatment i.e. not targeting the condition per se. Such usage included to assist with sleep disturbances or restlessness (anorexia nervosa). The use of certain antidepressants for the treatment of bulimic symptoms was also noted, as well as use of agents where co-morbid conditions existed that might benefit from primary pharmacological intervention e.g. anxiety disorders. This approach had been noted in an editorial (Szabo, 2001) where the emphasis was specifically on the use of so called "antidepressant" agents (in parenthesis as the same drugs are used to treat anxiety disorders). The potential to understand biology through response to intervention was raised, with a specific focus on the serotonergic neurotransmitter system. This theme was explored in an invited commentary on a chapter related to pharmacotherapy for eating disorders (Szabo, 2003). Whilst reiterating the role of pharmacological interventions it was specifically argued that on the basis of evidence, notwithstanding the use of serotonergic agents to target eating disorder symptoms, the preoccupation with the serotonergic system may need to be revisited - an understanding advanced in the earlier 1997 publication (Berk, Kessa, Szabo \& Butkow,1997).

\section{RESISTANCE TRAINING FOR HOSPITALIZED ANOREXICS}

The inclination of anorexia nervosa sufferers to engage in inappropriate aerobic exercise led to a series of papers arising from research that investigated the possibility that controlled resistance training i.e. non aerobic strength training might be a useful adjunct to in- patient treatment for anorexia nervosa (Szabo \& Green, 2002; Chantler, Szabo \& Green, 2006). At the time of undertaking the research that led to the subsequent, aforementioned, publications there was limited information on the incorporation of exercise into a treatment programme for anorexia nervosa (Beumont, Arthur, Russell \& Touyz, 1994; Thien, Thomas, Markin \& Birmingham, 2000). With the use of dumbbells (upper body) and therapeutic elastic bands for the lower body 2 groups of hospitalized anorexics were recruited with one participating in the supervised training program, the other not. Both groups improved over the duration of the training program both in relation to eating disorder and mood disorder related symptoms with no significant difference between the groups i.e. the training program was not prejudicial where implemented under supervision with controlled caloric intake(Szabo \& Green, 2002) . The subsequent paper (Chantler, Szabo \& Green, 2006) focused on the extent of strength improvement amongst those who participated in the program. It was clearly demonstrated that specific muscle 
groups improved, noting that adequate refeeding at the time of the program contributed to the strength benefits of the training program. At the time of the publication there was only one other study reporting on changes in muscular strength for hospitalized anorexics (Kratz \& Roessner, 2003). In essence the studies supported the incorporation of such an intervention into a treatment program, whilst also recommending areas for future research.

\section{OUTCOMES}

Whilst the inpatient program at Tara Hospital had been in existence for some 15 years at the time of a study on outcomes published in 1998 (Szabo \& Terre Blanche, 1998) no such data existed, from such a setting, albeit that clinical descriptions of anorexia nervosa within the South African populations had been published in the 1970's (George \& Smart, 1976; Norris, 1979). Within the South African context there were only 2 South African outcome studies, both from Groote Schuur Hospital in Cape Town, with neither study emanating from a dedicated eating disorders unit (Touyz \& Beumont, 1984; Nash \& Colbon, 1994 ). The study findings (Szabo \& Terre Blanche, 1998) emphasized that beyond weight restoration as a primary outcome, a number of related dimensions of both psychological and emotional functioning also improved with adequate weight restoration. Specifically it was found that nutritional status was positively correlated with mood related symptom improvement. This provided important data supporting a nutritional rather than a medication based approach to the treatment of mood symptoms in anorexia nervosa (Szabo, 2010). Moreover the study (Szabo \& Terre Blanche, 1998) also clearly demonstrated the range of improvements beyond weight restoration, thus providing data to refute the perception that admission was simply a weight gaining exercise - often cited as a basis for non- admission.

\section{COMPLICATIONS}

Anorexia nervosa is a multisystem disease insofar as all organ systems are impacted by the weight loss, yet with most anorexics remarkably resilient in terms of medical comorbidity. However, even on that basis one needs to be mindful. Two papers captured this issue in relation to tuberculosis, specifically amongst hospitalized anorexics (Szabo, Rausch \& Freeman, 1998; Szabo, 1998b). The diagnosis was unexpected, not least of all in a series of cases that highlighted risk factors for the possibility of comorbidity (Szabo, Rausch \& Freeman, 1998). This was followed by a special article (Szabo, 1998b) that looked at both similarities and differences between the conditions, and raised the issue of demographic stereotyping as a confounder in possible missed diagnosis. Given the high mortality rate amongst anorexics, which range from $6.6 \%$ at 10 year follow up (Eckert, Halmi, Marchi, Grove \& Crosby , 1995) to $15 \%$ at 20 year follow up Ratnasuriya, Eisler, Szmukler \& Russell, 1991) the need for awareness of a potentially life threatening comorbidity was critical. Specifically in a country with a high incidence of tuberculosis. Given the mortality rates amongst anorexia nervosa sufferers, it was suprising that the mechanism of death was so poorly understood. However, the sudden death of an anorexia sufferer prompted an autopsy (Derman \& Szabo, 2006). The result revealed pulmonary thrombo-emboli which had not been considered clinically. Review of the ante-mortem clinical findings noted a relative tachycardia which might have been a harbinger. However, the essence of the paper was that sudden death in anorexia nervosa sufferers warrants autopsy to assist in developing a risk profile. A series of cases of bulimia nervosa had been described where the patients had also presented with blood letting (Parkin \& Eagles, 1993). The authors interpreted the behavior as resulting from their bulimia nervosa i.e. a forme fruste of purging. In response and following review of the case material the suggestion was that each case appeared to suffer from borderline personality disorder where such behavior i.e. cutting/ self- mutilation would typically be a feature (Szabo, 1992). The issue was interpretation, but ultimately that such behavior potentially spoke to severity of illness (bulimia nervosa) and where anaemia was associated with bulimia nervosa to consider the presence of such behavior, and co-morbidity.

\section{CONCLUSION}

In essence, my work has been strongly influenced by clinical work in the field with consequent effort to contribute to clinician knowledge both locally and internationally. The work has contributed in some form to knowledge of risk and vulnerability, specifically within a South African and African context. In this respect the current paper provides a perspective based on my work, noting that comprehensive reviews of eating disorders in Africa, mentioned earlier, document the range of work undertaken in the field emanating from the continent. More recently (van Hoeken, Burns \& Hoek, 2016) it has been observed that "The epidemiological study of eating disorders in Africa is still in its infancy". The same review stated that "No cases of anorexia nervosa were reported in African epidemiological studies" whilst also noting, "The prevalence rate of bulimia nervosa in women in Africa is within the range reported for western populations, as well as African Americans and Latin Americans". Whilst acknowledging the paucity of studies providing epidemiological data, it is well to distinguish information derived from such data from the clinically documented experience. 
My journey has been important for me personally as well as for the understanding of eating disorders both in South Africa and internationally. It has been a journey whose path has been shaped to some extent by broader socio-political events in South Africa, not a routine experience. In working with eating disorders, I have been tested clinically and enriched as a clinician through the challenges that sufferers present. As difficult as eating disorder patients can be, their recovery is indeed highly rewarding not only to them and their families - but also to me. I consider my work in this field of psychiatry to have provided me with unique insights into the human psyche, and as such have a debt of gratitude to my patients for such an opportunity. It seems that no matter what else I have encountered as my career unfolded - eating disorders were constant in my work. I have shifted away from eating disorders research, but the knowledge and experience gained has contributed to my clinical work and served to refine my clinical acumen. However, of course, it is the patients themselves who have taught and accompanied me on this journey - which continues.

\section{ACKNOWLEDGEMENT}

Thanks to Peter Cleaton Jones for his mentoring

\section{CONFLICT OF INTEREST}

Nil/no other parties involved in contributing

\section{ETHICAL APPROVAL}

Not applicable as this is a clinical perspective and not a research study

\section{FUNDING}

$\mathrm{Nil} /$ no funding required

\section{INFORMED CONSENT}

Not applicable as this is a clinical perspective and not a research study

This paper is based on content that contributed to the award of a DSc in 2018

\section{REFERENCES}

Berk, M., Kessa, K., Szabo, C.P., \& Butkow, N. 1997. The augmented platelet intracellular calcium response to serotonin in Anorexia Nervosa but not Bulimia may be due to subsyndromal depression. International Journal of Eating Disorders, 22: 57-63.

Beumont, P.J., George, G.C.W., \& Smart, D.E. 1976. "Dieters" and "vomiters" and "purgers" in anorexia nervosa. Psychological Medicine, 6: 617-632.

Beumont, P., Arthur, B., Russell, J.D., \& Touyz, S.W. 1994. Excessive physical activity in dieting disorder patiemts: proposals for a supervised exercise program. The International Journal of Eating Disorders, 15: 21-36.

Blair, C., Freeman, C., \& Cull, A. 1995. The families of anorexia nervosa and cystic fibrosis patients. Psychological Medicine, 25:985-93

Buchan, T., Gregory, L.D. 1984. Anorexia Nervosa in a Black Zimbabwean. British Journal of Psychiatry, 145: 326-330.
Cameron, E.M., \& Ferraro, F.R. 2004. Body dissatisfaction in college women after exposure to magazine images. Perceptual and Motor Skills, 98: 1093-1099.

Chantler, I., Szabo, C.P., \& Green, K 2006. . Muscular strength changes in hospitalized anorexic patients after an eight week resistance training programme. International Journal of Sports Medicine, 28: 660-665.

Dare, C., Le Grange, D., Eisler, E., \& Rutherford, J. 1994. Redefining the psychosomatic family: family process of 26 eating disorder families. International Journal of Eating Disorders, 16: 211-226.

Delport, I., \& Szabo, C.P. 2008. Eating disorders in South Africa: an inter-ethnic comparison of admission psychometric data of hospitalised sufferers. (Scientific letter) South African Medical Journal, 98: 272-273

Derman, T., \& Szabo, C.P. 2006. Why do anorexics die ? A case of sudden death. International Journal of Eating Disorders, 39: $260-262$ 
Dolan, B. 1991. Cross-Cultural Aspects of Anorexia Nervosa and Bulimia: A Review. International Journal of Eating Disorders, 10: 67-78.

Eckert, E.D., Halmi, K.A., Marchi, P., Grove, W., \& Crosby, R. 1995. Ten-year follow-up of anorexia nervosa: clinical course and outcome. Psychological Medicine, 25:143-56

Fahy, T.A., Robinson, P.H., Russell, G.F.M., \& Sheinman, B. 1988. Anorexia Nervosa Following Torture in a Young African Woman. British Journal of Psychiatry, 153: 385-387.

Famuyiwa, 0.0. 1988. Anorexia nervosa in two Nigerians. Acta Psychiatrica Scandinavica, 78 : 550-554.

Field, A.E., Cheung, L., Wolf, A.M., Herzog, D.B., Gortmaker, S.L., \& Colditz, G.A. 1999. Exposure to the mass media and weight concerns among girls. Pediatrics, 103 : E36

Freeman, A., \& Szabo, C.P. 2005. Eating disorders in South African males: a review of the clinical presentation of hospitalised patients. South African Journal of Psychology, 35: 601-622.

Gabriel, B., \& Szabo, C.P. 2001. The demographics of hospitalised anorexics: a ten year review. (Scientific Letter) South African Medical Journal, 91:751-752.

Garner, D.M., Garfinkel, P.E., Schwartz, D., \& Thompson, M. 1980. Cultural expectations of thinness in women. Psychological Reports, 47: 483-491.

Hamilton, K., \& Waller, G. 1993. Media influences on body size estimation in anorexia and bulimia. An experimental study. British Journal of Psychiatry, 162:837-840.

Hitzroth, V., Wessels, C., Zungu-Dirwayi, N., Oosthuizen, P., \& Stein, D.J. 2001. Muscle Dysmorphia: A South African sample. Psychiatry and Clinical Neurosciences, 55: 521-523.

Hoek, H.W., Bartelds, A.I.M., Bosveld, J.J., van der Graff, Y., Limpens, V.E., Maiwald, M., \& Spaaij, C.J. 1995. Impact of Urbanisation on Detection Rates of Eating Disorders. American Journal of Psychiatry, 152: 1272-1278.

Hooper, M.S.H., \& Garner, D.M. 1986. Application of the Eating Disorders Inventory to a Sample of Black, White, and Mixed Race schoolgirls in Zimbabwe. International Journal of Eating Disorders, 5: 161-168.
Ilechukwu, S.T., \& Nhiwatiwa SM. Bulimia nervosa in an atypical setting: case report from Nigeria. British Journal of Psychiatry, 152: 864-865.

Kim, K.H. 2007. Religion, weight perception and weight control behaviour. Eating Behaviors, 8(1): 121- 131.

Kratz, 0., \& Roessner, V. 2003. Change in muscle strength in patients with anorexia nervosa during inpatient treatment - a pilot study. Zeitschrift Fur Kinder- Und Jugendpsychiatrie Und Psychotherapie, 31: 277-284

Lee, S. 1996. Reconsidering the status of anorexia nervosa as a western culture-bound syndrome. Social Science and Medicine, 42: 21-34.

le Grange, D., Tibbs, J., \& Noakes, T.D. 1994. Implications of a Diagnosis of Anorexia Nervosa in a Ballet School. International Journal of Eating Disorders, 15: 369-376.

Minuchin, S., Rosman, B.L., \& Baker, L. 1978. Psychosomatic Families: Anorexia Nervosa in Context. Cambridge, Massachusetts: Harvard University Press.

Morris, P.F., \& Szabo, C.P. 2013. Meanings of thinness and dysfunctional eating in black South African females: a qualitative study. African Journal of Psychiatry, 16: 338-342.

Mumford, D.B., Whitehouse, A.M., \& Platts, M. 1991. Sociocultural correlates of eating disorders among Asian schoolgirls in Bradford. British Journal of Psychiatry, 158: 222- 228.

Nash, E.S., \& Colborn, A.L. 1994. Outcome of hospitalised anorexics and bulimics in Cape Town, 1979-1989. South African Medical Journal, 84: 74-79.

Norris, D.L. 1979. Clinical diagnostic criteria for primary anorexia nervosa. South African Medical Journal, 56: 987-993.

Nwaefuna, A. 1981. Anorexia nervosa in a developing country. British Journal of Psychiatry,138: 270.

Parkin, J.R., \& Eagles, J.M. 1993. Blood-letting in bulimia nervosa. British Journal of Psychiatry, 162: 246-248.

Patton, G.C., Carlin, J.B., Shao, Q., Hibbert, M.E.; Rosier, M.; Selzer, R.; \& Bowes, G..1997. Adolescent Dieting: Healthy Weight Control or Borderline Eating Disorder? Journal of Child Psychology and Psychiatry, 38: 299-306. 
Pike, K.M., \& Rodin, J. 1991. Mothers, Daughters, and Disordered Eating. Journal of Abnormal Psychology, 100: 198-204.

Pinhas, L., Heinman, M., Bryden, P., Bradley, S., \& Toner, B. 2008. Disordered eating in Jewish adolescent girls. Canadian Journal of Psychiatry, 53(9): 601-608.

Pumariega, A. 1986. Acculturation and eating Attitudes in Adolescent Girls: A Comparative and Correlational Study. Journal of the American Academy of Child Psychiatry, 25: 276-279.

Ratnasuriya, R.H., Eisler, I., Szmukler, G.I., \& Russell, G.F.M. 1991. Anorexia Nervosa: Outcome and Prognostic Factors after 20 Years. British Journal of Psychiatry, 158: 495-502.

Selvini Palazzoli, M. 1974. Self Starvation: From the Intrapsychic to the Transpersonal Approach to Anorexia Nervosa. London: Chaucer.

Sykes, D.K., Leuser, B., Melia, M., Gross, M. 1988. A demographic analysis of 252 patients with anorexia nervosa and bulimia. International Journal of Psychosomatics, 35 (1-4): 5-9.

Srinivasagam, NM, Kaye, W.H., Plotnicov, K.H., Greeno, C., Weltzin, T. E., \& Rao, R. 1995. Persistent Perfectionism, Symmetry, and exactness After Long-Term Recovery From Anorexia Nervosa. American Journal of Psychiatry, 152 (11): 1630-1634.

Szabo, C.P., Berk, M., Tlou, E., \& Allwood, C.W. 1995. Eating disorders in black South African females: a series of cases. South African Medical Journal, 85 :588-590.

Szabo, C.P., \& Hollands, C. 1997a. Abnormal eating attitudes in secondary school girls in South Africa- a preliminary study. South African Medical Journal, 87 : 524-531.

Szabo, C.P., \& Hollands, C. 1997b. Factors influencing eating attitudes in secondary school girls in South Africa- a preliminary study. South African Medical Journal, 87: 531-534.

Szabo, C.P. 1992. Blood- letting in Bulimia Nervosa. (Letter) British Journal of Psychiatry, 162: 708.

Szabo, C.P. September 1994. Fat is not just a feminist issue. Playboy (South African Edition), 31.

Szabo, C.P. 1995. Management of eating disorders. Psychiatry in Practice, March/April: 13-16

Szabo, C.P. 1996. Playboy centrefolds and eating disordersfrom male pleasure to female pathology? South African Medical Journal, 86 (7) : 838-839.
Szabo, C.P. 1998a. Cross-cultural issues in eating disorders. Specialist Medicine, August issue: 13-15,25,32,38.

Szabo, C.P. 1998b. Tuberculosis and Anorexia nervosa (Special article). South African Medical Journal, 30: 275 - 276.

Szabo, C.P. 1999. Eating Attitudes in Black South Africans. (Letter) American Journal of Psychiatry, 156: 981-982.

Szabo, C.P. 2001. Antidepressants in the treatment of eating disorders. (Editorial) Journal of Depression and Anxiety, 4 : 1.

Szabo, C.P. 2002. Youth at risk: dieting and eating disorders. A South African perspective. (Editorial) South African Medical Journal, 92: 282-283.

Szabo, C.P. 2003. Eating disorders and pharmacotherapy. (Commentary) WPA series Evidence and Experience in PsychiatryVolume 6.Eating Disorders Editors: M Maj, K Halmi, J.J Lopez-Ibor \& N Sartorius. Wiley: Chichester, 310-312

Szabo, C.P. 2008. A case of male anorexia nervosa with Klinefelter's syndrome, 22 years later. British Journal of Psychiatry, 193:388.

Szabo, C.P. 2009. Eating Disorders. Reach Publishers, Wandsbeck, South Africa. 71 81, 103-109

Szabo, C.P. 2010. Complexity of treating eating disorders. Medical Chronicle, April: 78/ reprinted in Serenity 2011; March: 10-11

Szabo, C.P. 2011. Eating Disorders: an African Perspective. In Contemporary African Psychiatry: A Review of Theory, Practice and Research. Eds Ndetei DM \& Szabo CP. Acrodile Publishing Limited, Nairobi, Kenya. 239-262

Szabo, C.P., \& Allwood, C.W. 2004a. "A cross-cultural study of eating attitudes in adolescent South African females" World Psychiatry, 3: 41-44.

Szabo, C.P., \& Allwood, C.W. 2004b. Application of the Eating Attitudes Test (EAT-26) in a rural, Zulu speaking, adolescent population in South Africa. World Psychiatry, 3: 169-171.

Szabo, C.P., \& Allwood, C.W. 2006. Body figure preference in South African adolescent females. A cross cultural study. African Health Sciences, 6: 201-206.

Szabo, C.P., \& le Grange, D. 2001. Eating disorders and the politics of identity: the South African experience. In Eating Disorders and Cultures in Transition. Editors: Mervat Nasser, Melanie Katzman, Richard Gordon. Routledge Ltd, London, 24-39. 
Szabo, C.P., \& Terre Blanche, M.J. 1997. Perfectionism in Anorexia Nervosa. (Letter) American Journal of Psychiatry, 154 (1):132

Szabo, C.P., Goldin, J., \& le Grange, D. 1999. Application of the Family Relations Scale to a sample of anorexics, bulimics and non-psychiatric controls: a preliminary study. European Eating Disorders Review, 7: 37-46.

Szabo, C.P., \& Green, K. 2002. Hospitalised anorexics and resistance training: impact on body composition and psychological wellbeing. A preliminary study. The Journal of Eating and Weight Disorders, 7: 293-297.

Szabo, C.P., Rausch, G., \& Freeman, C.P. 1998. Hospitalised anorexics and Tuberculosis: a series of cases. European Eating Disorders Review, 6 : 171-177.

Szabo, C.P., \& Terre Blanche, M.J. 1998. Hospitalised anorexics: a preliminary evaluation of an inpatient programme. South African Medical Journal, 88: 312-318.

Szabo, C.P. 2015. Prevalence and Incidence of Eating Disorders in Underrepresented Countries In: The Wiley-Blackwell Handbook of Eating Disorders, Volume 1 (Smolak L \& Levine M, Eds.) and Volume 2 (Levine M \& Smolak L, Eds.) John Wiley \& Sons, LTD: Chichester, 79-92.

Thien, V., Thomas, A., Markin, D., \& Birmingham, C.L. 2000. Pilot study of a graded exercise program for anorexia nervosa. The International Journal of Eating Disorders, 28: 101-106

Touyz, S.W., \& Beumont, P.J.V. 1984. Anorexia nervosa. A follow up investigation. Medical Journal of Australia, 141: 219-222.

Visser, J., Notelovitz, T., Szabo, C.P., \& Fredericks, N. 2014. Abnormal eating attitudes and weight loss behaviours of adolescent girls attending a "traditional" Jewish high school in Johannesburg, South Africa. South African Journal of Clinical Nutrition, 27: 208-216.

van Hoeken, D., Burns, J.K., Hoek, H.W. 2016. Epidemiology of eating disorders in Africa. Current Opinion in Psychiatry, 29 (6):372-7

Wolfe, B.E., Metzger, E.D., Jimerson, D.C. 1997. Research Update on Serotonin Function in Bulimia Nervosa and Anorexia Nervosa. Psychopharmacology Bulletin, 33: 345-354. 
